\title{
Proceso de mejora continua en empresas procesadoras de plásticos de la Costa Oriental del Lago
}

\section{Continuous improvement process in plastic processing companies on the East Coast of Lake}

\author{
\Cinthia Castillo \\ cinthiagcc83@gmail.com \\ ORCID: 0000-0003-1305-2961 \\ Universidad del Zulia - Venezuela \\ Artículo recibido en enero 2019 / Arbitrado en febrero 2019 / Publicado en julio 2019
}

RESUMEN

La investigación tuvo como propósito analizar el proceso de mejora continua presente en la gestión de calidad de las empresas procesadoras de plásticos de la Costa Oriental del Lago. La metodología fue de tipo descriptiva, bajo un diseño no experimental, de campo y transeccional. La población quedó conformada por tres (3) empresas procesadoras de plásticos ubicadas en la Costa Oriental del Lago. Para la recolección de datos se empleó la encuesta mediante un cuestionario, el mismo quedó conformado por 20 ítems con escala dicotómica. La validez se realizó a través del juicio de expertos, y para calcular su confiabilidad se empleó el coeficiente de KüderRichardson, obteniéndose 0,923. El análisis de datos se realizó mediante la estadística descriptiva, con base en las frecuencias relativas. Se concluye mediana presencia del proceso de mejora continua, dentro de la gestión de calidad, a través de planificar, hacer o ejecutar, verificar y actuar.

Palabras clave: Actuar, gestión de calidad, hacer o ejecutar, planificar, proceso de mejora continua, verificar

ABSTRACT

The purpose of the research was to analyze the continuous improvement process present in the quality management of plastics processing companies on the Eastern Lake Coast. The methodology was descriptive, under a non-experimental, field and transectional design. The population was made up of three (3) plastics processing companies located on the eastern shore of the lake. The survey was used to collect data using a questionnaire, which was made up of 20 items with a dichotomous scale. Validity was carried out through expert judgment, and the Küder-Richardson coefficient was used to calculate its reliability, obtaining 0.923. Data analysis was performed using descriptive statistics, based on relative frequencies. Medium presence of the continuous improvement process is concluded, within quality management, through planning, doing or executing, verifying and acting.

Keywords: Act, quality management, do or execute, plan, continuous improvement process, vÆNTRODUCCIÓN 
Los constantes cambios que circundan las organizaciones empresariales del siglo XXI, y por consiguiente, a las exigencias del mercado laboral en el cual éstas se encuentran inmersas, demandan sistemas, métodos, metodologías y procesos que garanticen su competitividad ante la globalización industrial. Asimismo, soportando los avances tecnológicos, adaptación del talento humano, concreción de políticas internas, objetivos, metas y ambiente, e inclusive las fusiones con otras empresas.

Visto así, según Cantú (2011), países de América Latina, entre ellos México, Colombia, Argentina, entre otros, consideran que la clave del éxito reside en ser competitivos, entendiendo por éxito que la sociedad obtenga satisfacciones tanto en lo material como en lo emocional, permitiéndoles vivir con calidad, promoviendo así el desarrollo de organizaciones competentes como base de una economía fuerte y sólida. De igual manera Cantú (2011:3) indica lo siguiente:

Los países de Latinoamérica necesitan una mayor cantidad de personas con preparación de calidad que desarrollen y operen organizaciones altamente competitivas que puedan incursionar exitosamente en los mercados mundiales en busca de la satisfacción que la sociedad demanda y merece.

Por lo tanto, es muy importante que la organización cuente con un personal capacitado con perfil de liderazgo, capaz de resolver situaciones inesperadas y tomar el control del rumbo para la cual fue estructurada. De igual forma, son un recurso estratégico como un sistema de compromiso que organizan y desarrollan procesos en función de una mejora continua. Así mismo, cumple un papel predominante en la toma de decisiones a fin de mejorar de forma continua los productos y/o servicios. Así como también, desarrollar procesos gerenciales exitosos, que les permita la capacidad para definir, alcanzar y evaluar los objetivos con el uso adecuado de sus recursos y la coordinación de todas las actividades que la conforman.
Al respecto, Miranda y otros (2007) definen la gestión como un conjunto de operaciones o acciones que dan vida y dinamismo a los periodos de actividades de una empresa. Debe ser organizada e integrada con las etapas clásicas del proceso administrativo que incluye previsión, planificación, organización, integración, dirección y control, es decir, la gestión es la acción que se ejecuta para llevar a cabo todas y cada una de las tareas del proceso administrativo-operativo con calidad en sus diferentes etapas.

De igual forma, la gestión es de gran importancia según lo establecido en la Norma ISO 9001:2008, la cual se refiere a las actividades coordinadas para dirigir y controlar una organización en la aplicación de los procesos de gestión, siendo de gran medida resultado importante del uso de la información como recurso estratégico para las empresas, bien sea desde la perspectiva de sus activos tangibles como recursos materiales, financieros y activos intangibles en conocimiento e información.

Por otra parte, retomando las ideas expuestas en la Norma ISO 9001:2008, la gestión de calidad es considerada como actividades coordinadas de una organización, la cual es una estrategia orientada a crear conciencia en todos los procesos organizacionales y las personas que trabajan en ella, manteniendo una mejora continua, con el objetivo de lograr la calidad óptima en todas las áreas.

En consonancia con esto, resulta importante reconocer el valor que recae sobre el talento humano en su entorno laboral, la calidad asumida y el trabajo bien realizado como respuesta a esa responsabilidad, pero también como fuente de satisfacción personal en donde la participación, compromiso y trabajo colaborativo son elementos fundamentales de una organización exitosa. El reconocimiento, como signo de humanidad del que lo otorga y del que lo recibe, definen este trasfondo ético que es característico de la gestión de calidad y expresa, en gran medida, el óptimo funcionamiento de aquellas que lo asumen como propio. 
Visto desde este enfoque, Cantú (2011) señala que, la gestión de calidad es una metodología fundamental que se aproxima a la existencia de procedimientos de autocorrección, basados en informaciones empíricas medibles que tienen como finalidad la mejora continua, mejor conocida como ciclo de Deming. Quizás por ello se puede afirmar que, en una organizaciónde calidad, los empleados trabajan como científicos y es que ese modo de hacer permite explicar el progreso tanto de las ciencias experimentales como de las empresas que aplican programas de calidad.

Por tal razón, la gestión de la calidad se ha convertido en el mundo moderno de hoy en una necesidad inevitable para que una organización pueda permanecer en el mercado. Sin embargo, los sistemas del mismo basados en las normas ISO, reflejan el consenso internacional en este tema, cobrando una gran popularidad $\mathrm{y}$, muchas han decidido tomar el camino de implantarlo.

Es por ello, que Udaondo (2007) define la gestión de calidad como un conjunto de caminos mediante los cuales se logra la calidad, incorporándolo al proceso de gestión, donde se dirigen y coordinan todas las actividades, se implantan los programas y se controlan los resultados con vistas a una mejora continua de sus procesos. Newell (2004), afirma que este proceso se orienta más, que en el pasado, hacia pequeñas mejoras incrementales permanentes y a puntos de inspección múltiples. Afirma que uno de los principales cambios de actitud con respecto a la calidad es que todo el mundo es responsable por la calidad, esto ha permitido muchos más puntos de inspección y, produce que las correcciones se hagan antes de que se requiera más trabajo adicional.

Newell (2004), además plantea como características de la gestión de la calidad los siguientes aspectos: un componente importante de la gestión moderna de la calidad es la orientación al cliente o al consumidor. El cliente, está interesado en un producto o servicio desde el punto de vista de su adecuación al uso, la garantía de que él recibirá los bienes o servicios que justifican lo que pagó por ellos y, la sensación que tiene después de recibir el producto o el servicio. Otra característica de la gestión moderna de la calidad es que considera las pequeñas mejoras incrementales como el enfoque adecuado para mejorar la calidad.

Explica este autor, el enfoque del Total Quality Management (TQM), Gestión de calidad Total, tiene un número considerable de prácticas formalizadas para introducir procesos de cambios pequeños, paso a paso, en el ciclo operativo normal de la organización. Aún más, hay sistemas especiales constituidos que permiten a todos los participantes en cualquier proceso sugerir cambios para mejorar la calidad de los procesos y productos. En este contexto, Newell (2004) explica que el enfoque del TQM debe estar planteado bajo el proceso o ciclo de mejora continua: planificar, hacer, controlar, actuar, para incrementar las mejoras en sus procesos.

Así las cosas, para evaluar la eficacia y eficiencia de los procesos se emplea frecuentemente el ciclo de Deming o PHVA (planificar, hacer, verificar y actuar) donde, según la Norma ISO 9001: 2008:

- En la fase "P" se establecen los objetivos y los procesos, de acuerdo con los requerimientos y políticas de la organización.

- En la fase "H" se ejecuta aquello que se planificó.

- En la fase "V" se siguen y evalúan los procesos y resultados.

- En la fase "A" se desarrollan acciones para mejorar el proceso, en este caso, el término apropiado que se sugiere asignar es el de rehacer o ajustar porque realmente es lo que se realiza en este paso.

De igual forma, la referida norma internacional, establece que es un sistema para dirigir y controlar una organización; por tanto, según esta norma, el hablar del sistema de gestión de calidad es referirse a la forma como una organización realiza la gestión empresarial asociada con la calidad. En términos generales, consta de la estructura organizacional junto con la documentación, procesos y recursos empleados para alcanzar sus objetivos y cumplir con los requisitos del cliente. 
Ahora bien, en Venezuela la gestión de calidad se ha caracterizado por procesos de globalización y competencia como respuesta a los inmutables avances nacionales e internacionales tanto en el sector tecnológico como industrial, lo que hace que en cada organización se aumente el nivel de complejidad en cuanto a los procedimientos administrativos que cada una de ellas posee, así mismo establecer los mecanismos necesarios que permitan garantizar los derechos de las personas.

Según la Gaceta oficial № 3742 Ley del Sistema Venezolano para la Calidad, la ISO permite fomentar las normas, reglamentaciones técnicas y procedimientos de evaluación, para facilitar la disponibilidad de bienes y servicios con calidad en el país, así como el óptimo funcionamiento del sistema para la gestión de la calidad; buscando estimular la calidad, competitividad del estado y de las empresas. De esta manera, se estaría promoviendo y asegurando la participación de todos los interesados en el funcionamiento del mismo. Así, como mecanismo para regular y controlar el mejoramiento continuo de las actividades del sistema venezolano que se realizan en el campo fortaleciendo así los lazos de confianza entre las partes involucradas.

Desde esta perspectiva, las fábricas procesadoras de plásticos tienen como misión diseñar y promover el mejoramiento continuo de los procesos en materia de calidad, promoviendo el mejoramiento continuo de los procesos asociados. De igual forma, garantizando el manejo de la gestión con sentido de negocio, basados en el desarrollo del personal y tecnología, con el mayor grado de seguridad, protección ambiental, calidad y flexibilidad, dirigidos hacia la máxima satisfacción de los clientes y búsqueda permanente de la excelencia, es decir, satisfacer las necesidades de la sociedad.

En este sentido, estas fábricas cuentan con una gerencia de la calidad, cuya función es dirigir los procesos de planificación, gestión y control del sistema de gestión de la calidad, mediante el desarrollo y establecimiento de políticas, directrices y métodos de trabajo. Esto permite la promoción de actividades de normalización y certificación de la calidad, con el propósito de optimizar el desempeño general de la organización, lograr la mejora continua e integral de sus procesos y la sustentabilidad.

Contextualizando la problemática, se tiene que dichas fábricas cuentan con diversos departamentos, entre estos recursos humanos, el cual también se encuentra en un proceso de implantación del sistema, teniendo como misión, ser una organización de servicios con asesorías en todos sus procesos. Asimismo, dicho departamento persigue dar respuesta oportuna, mediante la aplicación de leyes, normas y procedimientos internos, asegurando un servicio eficiente en la gestión social con la formación y desarrollo del talento humano, a fin de satisfacer las necesidades en las organizaciones para así, contribuir con la productividad y continuidad operacional.

En este contexto, en los departamentos de control de calidad de las fábricas procesadoras de plástico de la Costa Oriental del Lago, según entrevista realizada a sus gerentes, es notoria la probable inexistencia de unificación de criterios en formas, procesos, lineamientos, normas, procedimientos, manejo de situaciones y controles administrativos. Por tal motivo, existe quizás dificultad al momento de auditar cada una de las fábricas en lo que respecta a sus procesos.

Asimismo, la inexistencia de estatutos y procedimientos establecidos en relación a la autogestión en estas procesadoras, no ayudan a solventar distintas actividades internas que satisfacen a la comunidad en general. Del mismo modo, carencias de recursos en material, debido a las requisiciones o solicitudes generadas que dependen del departamento de compras en donde las compras deben hacerse con un año de anticipación, para que puedan ser consolidadas en el año en curso de cada periodo.

En consonancia a lo antes planteado, según observaciones directas realizadas en sitio, se ha evidenciado que estas fábricas ameritan normas, lineamientos y procedimientos administrativos orientados a la consolidación de los criterios de calidad que garanticen el funcionamiento eficiente, eficaz e idóneo de los procesos de fabricación. Sin 
embargo, la gerencia de calidad se encuentra en una etapa de planificación estratégica dependiente del departamento de calidad, el cual ha logrado el establecimiento de políticas, misión, visión, alcance y objetivos de dicha organización, para de tal manera encaminarse a la implementación de los estándares de calidad que se ameritan hoy en día con relación a los servicios prestados.

En líneas generales, se tiene que la gestión de calidad busca orientar el trabajo diario hacia la calidad permanente sea cual sea la naturaleza de la organización en la cual se pretenda implementar la normativa ISO. Además, priorizando los procesos y servicios con el fin de obtener la mejora continua para satisfacer las necesidades y expectativas de sus clientes externos e internos, logrando reducir variabilidad en los procesos, reducir pérdidas de tiempo y desperdicios a fin de mejorar y corregir los problemas potenciales, lo que conlleva a que tenga eficiencia en todos sus recursos. La habilidad de estas procesadoras, para adoptar nuevos esquemas de gestión de calidad será lo que las impulse a situarse entre las empresas más rentables a nivel mundial.

Por consiguiente, se hace necesario en las fábricas procesadoras de plástico de la Costa Oriental del Lago, analizar cómo es el proceso de mejora continua presente en la gestión de calidad, lo cual redundará en dar confianza a sus clientes actuales, como potenciales. Del mismo modo, cumplir con sus expectativas, mejorando así el rendimiento, coordinación y productividad de las actividades, logrando mantener consistentemente las expectativas explicitas e implícitas, tanto sobre sus usuarios como los servicios generados en las referidas organizaciones, cuando se requiera tomar decisiones.

\section{MATERIALES Y METODO}

La metodología aplicada fue de tipo descriptiva, bajo un diseño no experimental, de campo y transeccional. La población quedó conformada por las tres (3) empresas procesadoras de plásticos ubicadas en la Costa Oriental del Lago, siendo los sujetos informantes gerentes generales, de operaciones, supervisores e inspectores de calidad, con un total de 15 sujetos encuestados.

Para la recolección de datos se empleó la encuesta mediante la aplicación de un cuestionario, el mismo quedó conformado por 20 ítems con escala dicotómica. La validez se realizó a través del juicio de expertos, y para calcular su confiabilidad se empleó el método del coeficiente de KüderRichardson, donde se obtuvo como valor de confiabilidad total del instrumento 0,923, al incluir todos los ítems. El análisis de datos se realizó mediante la estadística descriptiva, con base en las frecuencias relativas, para tal efecto, la investigadora diseñó un (1) baremo, mostrado en el cuadro 1 .

Cuadro l. Baremo de interpretación de la frecuencia

\begin{aligned} & \hline RANGO PARA FRECUENCIA RELATIVA \\ & \hline SI $\geq 70 \%$ Alta presencia / Alta fortaleza \\ & $40 \% \leq \mathrm{SI}<70 \%$ Mediana presencia / Leve fortaleza \\ & $\mathrm{SI}<40 \%$ Baja presencia / Debilidad \\ & \hline\end{aligned}

Fuente:Elaboración propia (2020) 


\section{RESULTADOS Y DISCUSION}

En la tabla 1 se muestran los resultados para el indicador planificar, de la dimensión denominada proceso de mejora continua. De acuerdo al baremo establecido, en las empresas procesadoras de plásticos de la Costa Oriental del Lago, se tiene mediana presencia de la planificación como parte del proceso de mejora continua en la gestión de calidad que desarrollan, situación que se devela al ubicarse en promedio el 68\% de respuestas en la opción sí, confiriéndole una leve fortaleza a la gestión.
Estos resultados reflejan que, según los encuestados, en estas empresas se da alta presencia a los procesos vinculados a: si la gerencia de calidad establece los objetivos de acuerdo a sus políticas (80\%), los objetivos de calidad definen las metas planteadas por la organización $(73,33 \%)$, y la planificación de calidad se enfoca a diseñar procesos que satisfagan las necesidades de los clientes (86,67\%), constituyendo esas acciones en altas fortalezas de su gestión de calidad.

Tabla 1. Indicador: Planificar

En la empresa donde usted trabaja:

1. ¿La gerencia de calidad establece los objetivos de acuerdo a sus políticas?

2. iLos objetivos de calidad definen las metas planteadas por la organización?

3. ¿La misión definida se adecua a la toma de decisiones?

4. ¿La planificación de calidad se enfoca a diseñar procesos que satisfagan las necesidades de los clientes?

5. ¿A partir de la planificación de la calidad, se puede hacer un control preciso de cada paso dentro del proceso de la gestión de la misma?

\section{ITEMS} INDICADOR

\begin{tabular}{|c|c|c|c|c|c|c|c|c|c|c|c|c|}
\hline $\mathrm{N}^{\circ}$ & $\mathrm{Si}$ & $\%$ & No & $\%$ & $\mathrm{n}$ & Cat & ría & $\mathrm{Si}$ & $\%$ & No & $\%$ & $\mathrm{n}$ \\
\hline 1 & 12 & 80 & 3 & 20 & 15 & Alta & $\mathrm{AF}$ & 10,20 & 68,00 & 4,80 & 32,00 & 15 \\
\hline 2 & 11 & 73,33 & 4 & 26,67 & 15 & Alta & $\mathrm{AF}$ & \multicolumn{5}{|c|}{ CATEGORÍA } \\
\hline 3 & 7 & 46,67 & 8 & 53,33 & 15 & Med. & LF & \multirow{3}{*}{\multicolumn{5}{|c|}{$\begin{array}{l}\text { MEDIANA PRESENCIA } \\
\text { LEVE FORTALEZA }\end{array}$}} \\
\hline 4 & 13 & 86,67 & 2 & 13,33 & 15 & Alta & $\mathrm{AF}$ & & & & & \\
\hline 5 & 8 & 53,33 & 7 & 46,67 & 15 & Med. & LF & & & & & \\
\hline
\end{tabular}

Fuente: Elaboración propia (2020)

Mientras, se evidencia mediana presencia en lo relacionado a si la misión definida se adecua a la toma de decisiones (46,67\%), y si a partir de la planificación de la calidad se puede hacer un control preciso de cada paso dentro del proceso de la gestión de la misma (53,33\%), viéndolas como leves fortalezas de su gestión.

Dado los resultados mostrados, se evidencia mediana congruencia con lo planteado en la teoría por Pérez y Munera (2007), quienes explican que, el planificar será mucho más fácil si se definen los objetivos de lo que se quiere alcanzar, y es más fácil también pues se puede verificar si lo que se hizo corresponde a lo planeado. Cabe destacar que, una mala planificación no permite una buena ejecución y tampoco una correcta verificación. Por lo tanto, la etapa de planificación es fundamental.

También establecen mediana coincidencia con la investigadora cuando asegura que, se debe realizar una efectiva planificación donde se conozcan los objetivos de una manera clara, donde se puede hacer un control preciso de cada paso dentro del proceso de la gestión de la calidad de la organización. 
En la tabla 2, se muestran los valores obtenidos de las respuestas correspondientes al indicador hacer o ejecutar. Se observa como el $68 \%$ de los sujetos encuestados respondieron de manera afirmativa a las proposiciones emitidas, indicando que las actividades relacionadas a los ítems se realizan con mediana presencia, de acuerdo al baremo utilizado para tal fin.

Tabla 2. Indicador: Hacer o ejecutar

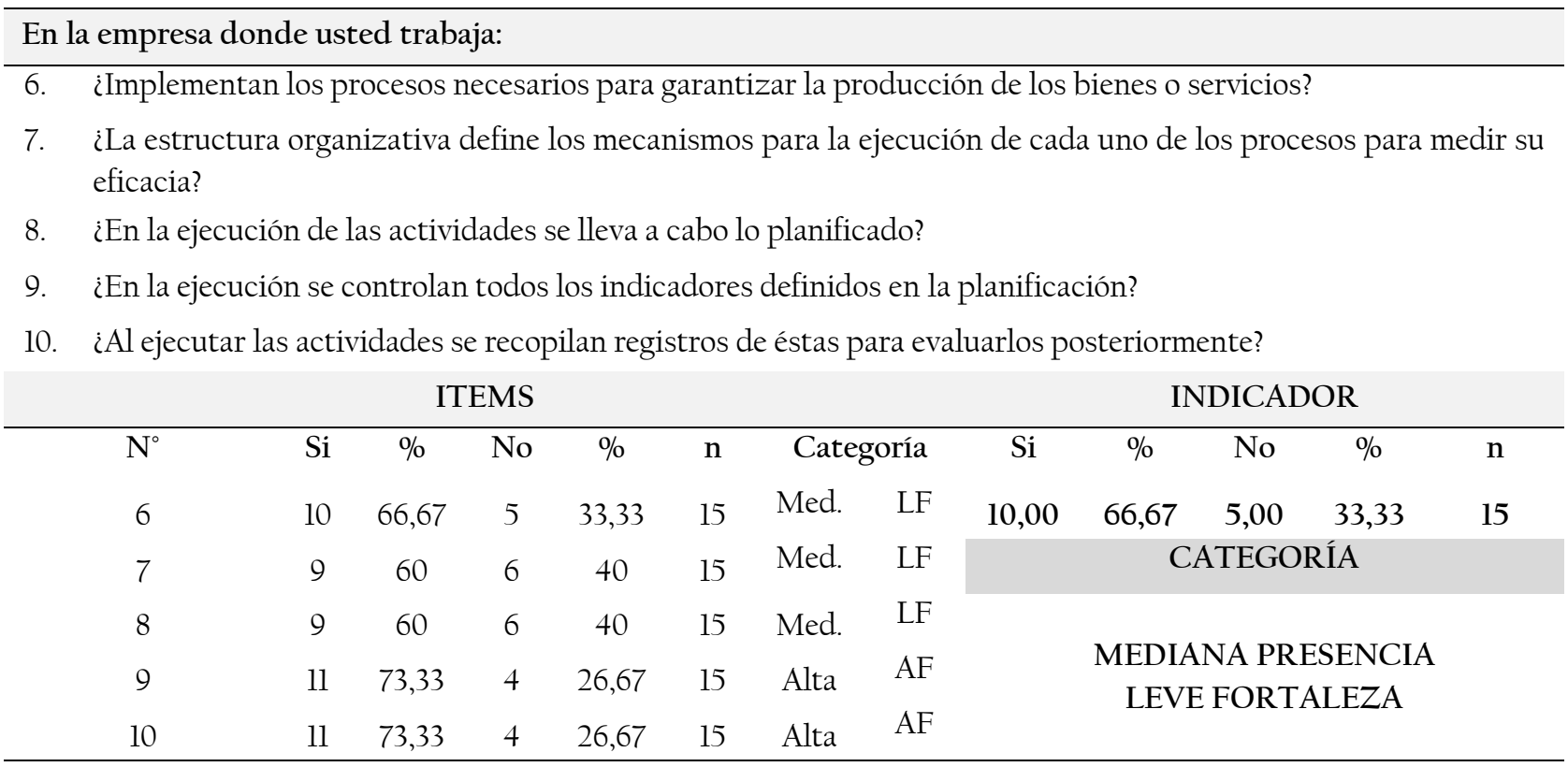

Fuente: Elaboración propia (2020)

Al detallar estos resultados se aprecia que, para el $73,33 \%$ de los encuestados se tiene alta presencia de que en la ejecución se controlen todos los indicadores definidos en la planificación, y al ejecutar actividades se recopilen registros de éstas para evaluarlos posteriormente, otorgándoles altas fortalezas en estos aspectos.

Mientras los ítems 6, 7 y 8 se catalogaron con mediana presencia, siendo leves fortalezas de la gestión, al obtener resultados de $66,67 \%$ y $60 \%$ (igual para ambos casos), de respuestas en la opción afirmativa, indicando según el baremo que, en estas empresas implementan los procesos necesarios para garantizar la producción de los bienes o servicios, la estructura organizativa define los mecanismos para la ejecución de cada uno de los procesos para medir su eficacia y en la ejecución de las actividades se lleva a cabo lo planificado.

Los resultados validan, medianamente, lo expuesto en la teoría que soporta la investigación, como es el caso de Muñoz (2009) para quien en esta etapa se tiene que llevar a cabo todas las acciones planificadas, estableciendo una correcta supervisión. Además, en esta fase se deben observar las posibles deficiencias del plan que se hayan encontrado y necesidades de formación descubiertas.

También validan, de forma moderada, lo afirmado por la investigadora para quien, en esta etapa se ejecuta el plan estratégico, lo que contempla: organizar, dirigir, asignar recursos y supervisar la ejecución, mientras se recopilan datos para verificarlos y evaluarlos en los siguientes pasos, es decir, en este paso se llevan a cabo los procesos ya definidos y se documentan las acciones realizadas para facilitar el desarrollo de las siguientes fases del ciclo. En pocas palabras, como lo dice la norma se hace o ejecuta aquello que se planificó. 
La tabla 3, muestra las respuestas correspondientes al indicador verificar, en la cual se aprecia como para el $64 \%$ de los encuestados, en promedio, en las empresas bajo estudio se tiene alta presencia de las actividades relacionadas al indicador. Esta situación es explicada por el comportamiento de las respuestas afirmativas dadas a cada ítem involucrado, las cuales arriban a la categoría de alta y mediana presencia.

Tabla 3. Indicador: Verificar

En la empresa donde usted trabaja::

1l. ¿Poseen la documentación de todas las tareas que en ésta se ejecutan?

12. ¿Las normas que rigen las actividades desempeñadas permiten que éstas sean eficientes?

13. ¿Se comparan los resultados esperados con los obtenidos?

14. ¿Comprueban que las actividades realizadas corresponden a lo planeado?

15. ¿Realizan procesos de monitoreo de la implementación del plan de ejecución, documentando las conclusiones?

\begin{tabular}{ccccccccccccc}
\multicolumn{1}{c}{ ITEMS } & \multicolumn{1}{c}{ INDICADOR } \\
\hline $\mathbf{N}^{\circ}$ & Si & $\%$ & No & $\%$ & n & Categoría & Si & $\%$ & No & $\%$ & $\mathbf{n}$ \\
11 & 11 & 73,33 & 4 & 26,67 & 15 & Alta & AF & 9,60 & 64,00 & 5,40 & 36,00 & 15 \\
12 & 10 & 66,67 & 5 & 33,33 & 15 & Med. & LF & \multicolumn{3}{c}{ CATEGORÍA } \\
13 & 10 & 66,67 & 5 & 33,33 & 15 & Med. & LF & & & \\
14 & 11 & 73,33 & 4 & 26,67 & 15 & Alta & AF & & MEDIANA PRESENCIA & \\
15 & 6 & 40,00 & 9 & 60,00 & 15 & Med. & LF & & LEVE FORTALEZA & \\
\hline
\end{tabular}

Fuente: Elaboración propia (2020)

De tal forma en las empresas estudiadas: poseen la documentación de todas las tareas que en ésta se ejecutan, y comprueban que las actividades realizadas corresponden a lo planeado, según el $73,33 \%$ de los sujetos encuestados, confiriéndole altas fortalezas a su gestión de calidad en estos aspectos.

Además, se evidencia mediana presencia de las siguientes actividades: las normas que rigen las actividades desempeñadas permiten que éstas sean eficientes $(66,67 \%)$; se comparan los resultados esperados con los obtenidos (66,67\%); y realizan procesos de monitoreo de la implementación del plan de ejecución documentando las conclusiones (40\%); delineándolas como leves fortalezas de su gestión.

Los resultados concuerdan, medianamente, con lo establecido por Pérez y Munera (2007), para quienes la verificación continúa luego de la etapa de ejecución, es decir, comprobar que lo que se hizo corresponde a lo planeado. De acuerdo con los autores, en esta fase se dan acciones de visualizar el resultado bien sea del servicio o del producto con respecto a lo que estaba planificado. Asimismo, validan de manera mediana lo considerado por la investigadora cuando afirma que, la verificación es el proceso de monitoreo de la implementación y evaluación del plan de ejecución, documentando las conclusiones. Se deduce que pasado un periodo previsto, los datos de control recopilados y analizados, se comparan con los requisitos especificados inicialmente, para saber si se han cumplido y, en su caso, evaluar si se ha producido la mejora esperada.

De seguido, en la tabla 4 , se muestran los resultados obtenidos para el indicador actuar, último indicador considerado para medir la dimensión proceso de mejora continua. Como se evidencia, el 64\% de las respuestas se posicionaron en la opción afirmativa, otorgándole a este indicador la categoría de mediana presencia, indicándose como una leve fortaleza de la gestión de calidad llevada a cabo. Al detalle se visualiza como solo el ítem 20 arriba a la categoría de alta 
presencia, por ende es una alta fortaleza de la gestión, con un promedio del 73,33\% en la opción si, en cuanto a si el personal conoce la política de calidad establecida por la empresa.

No obstante, el resto de los ítems ostentaron mediana presencia, observándose como para el $46,67 \%$ de los sujetos encuestados en el quehacer diario realizan pequeñas mejoras que aumenten la productividad; y para el 66,67\%: se documentan los procesos para ofrecer retroalimentación en la mejora de la fase de planificación; las estrategias son comunicadas a los departamentos correspondientes; y los líderes motivan a sus empleados para que sean participativos.

Tabla 4. Indicador: Actuar

\begin{tabular}{|c|c|c|c|c|c|c|c|c|c|c|c|}
\hline \multicolumn{12}{|c|}{ En la empresa donde usted trabaja:: } \\
\hline \multicolumn{12}{|c|}{ 16. ¿En el quehacer diario realizan pequeñas mejoras que aumenten la productividad? } \\
\hline \multicolumn{12}{|c|}{ 17. ¿Se documentan los procesos para ofrecer retroalimentación en la mejora de la fase de planificación? } \\
\hline \multicolumn{12}{|c|}{ 18. ¿Las estrategias son comunicadas a los departamentos correspondientes? } \\
\hline \multicolumn{12}{|c|}{ 19. ¿Los líderes motivan as } \\
\hline \multicolumn{12}{|c|}{1 personal conoce la política de calidad establecid } \\
\hline \multicolumn{7}{|c|}{ ITEMS } & \multicolumn{5}{|c|}{ INDICADOR } \\
\hline $\mathrm{N}^{\circ}$ & $\mathrm{Si}$ & $\%$ & No & $\%$ & $\mathrm{n}$ & Categoría & Si & $\%$ & No & $\%$ & $\mathrm{n}$ \\
\hline 16 & 7 & 46,67 & 8 & 53,33 & 15 & Med. LF & 9,60 & 64,00 & 5,40 & 36,00 & 15 \\
\hline 17 & 10 & 66,67 & 5 & 33,33 & 15 & Med. & \multicolumn{5}{|c|}{ CATEGORÍA } \\
\hline 18 & 10 & 66,67 & 5 & 33,33 & 15 & Med. LF & \multirow{3}{*}{\multicolumn{4}{|c|}{$\begin{array}{l}\text { MEDIANA PRESENCIA } \\
\text { LEVE FORTALEZA }\end{array}$}} & \\
\hline 19 & 10 & 66,67 & 5 & 33,33 & 15 & Med. & & & & & \\
\hline 20 & 11 & 73,33 & 4 & 26,67 & 15 & Alta & & & & & \\
\hline
\end{tabular}

Fuente: Elaboración propia (2020)

Estos resultados logran validar, de forma mediana, la teoría expuesta por Muñoz (2009) para quien, en este paso del ciclo el movimiento debe perpetuarse mediante la fase de estandarización y ajuste en la que el proceso se asiente en la organización y forme parte de su funcionamiento habitual. Es decir, la mejora de la atención al cliente puede abrir nuevas vías de actuación, creciendo la necesidad de abrir nuevas vías de comunicación o introducción de mejoras tecnológicas que apoyen la acción de los técnicos, y que pueden necesitar la creación de nuevos planes de acción. En el quehacer diario se pueden hacer pequeñas mejoras que aumenten la productividad.

También alcanzan mediana congruencia con lo expresado por la investigadora, cuando deduce que, en la fase actuar, dentro del ciclo de mejora continua, se deben analizar y corregir desviaciones detectadas así como proponer mejoras a los procesos utilizados. En esta fase se supone la puesta en práctica de las modificaciones detectadas en la fase anterior, con la finalidad de establecer acciones correctivas, disminuyendo la diferencia entre las necesidades del cliente y la ejecución del proceso. Esta fase retroalimenta la planificación para optimizar las variables manipulables del proceso.

Para la dimensión proceso de mejora continua, cuyos resultados se concentran en la tabla 5 , se observa cómo el 65,67\%, en promedio, de los encuestados consideraron la opción afirmativa, indicando mediana presencia del proceso de mejora continua, dentro de la gestión de calidad que realizan las empresas procesadoras de plásticos de la Costa Oriental del Lago, delineando este aspecto como leve fortaleza de su gestión. 
Tabla 5. Dimensión: Proceso de mejora continua

\begin{tabular}{|c|c|c|c|c|c|c|}
\hline INDICADOR & & $\mathrm{Si}$ & $\%$ & No & $\%$ & BAREMO \\
\hline Planificar & & 10,20 & 68,00 & 4,80 & 32,00 & Mediana presencia / Leve fortaleza \\
\hline Hacer o ejecutar & & 10,00 & 66,67 & 5,00 & 33,33 & Mediana presencia / Leve fortaleza \\
\hline Verificar & & 9,60 & 64,00 & 5,40 & 36,00 & Mediana presencia / Leve fortaleza \\
\hline \multirow[t]{2}{*}{ Actuar } & & 9,60 & 64,00 & 5,40 & 36,00 & Mediana presencia / Leve fortaleza \\
\hline & Totales & 9,85 & 65,67 & 5,15 & 34,33 & Mediana presencia / Leve fortaleza \\
\hline
\end{tabular}

Fuente: Elaboración propia (2020)

Al detalle, se puede apreciar que todas las fases del proceso de mejora continua fueron catalogadas con mediana presencia: planificar $(68 \%)$; hacer o ejecutar (66,67\%); verificar (64\%); y actuar (64\%); delineándolas como leves fortalezas de la gestión y convirtiéndolas en oportunidades de mejora, según el criterio de la investigadora.

Estos resultados logran validar, medianamente, los postulados de Pérez y Munera (2007), para quienes el ciclo (PHVA) es un proceso esencial, que debe ser realizado en cada una de las etapas, en cada una de las acciones a realizar. Para ello, se planifica lo que se va a hacer, luego se hace, se verifica si lo que se hace corresponde a lo planeado para actuar de dos maneras, actuar para corregir si no correspondió a lo planeado y actuar para mejorar si correspondió a lo planeado.

También alcanzan mediana congruencia con lo expresado por la investigadora, cuando afirma que las empresas deben establecer procesos para asegurarse de que el seguimiento se realice de manera coherente con los requisitos de seguimiento y mejora establecidos en la Norma ISO. Al mismo tiempo, dado los resultados de su proceso de seguimiento establecer las acciones correctivas y preventivas, según se ameriten. Por ello, el proceso de mejora continua, en la gestión de calidad, debe ser un ciclo cuyas fases (planificar, hacer, verificar y actuar) permitan la mejora de los procesos y la retroalimentación de la gestión.

\section{CONCLUSIONES}

Se analizó el proceso de mejora continua presente en la gestión de calidad de las empresas procesadoras de plásticos de la Costa Oriental del
Lago, concluyendo que estas empresas poseen mediana presencia de un conjunto de acciones dirigidas a obtener la mayor calidad posible de sus productos, delineando este aspecto como leve fortaleza de su gestión.

Debe mencionarse que dada esta categoría de mediana presencia alcanzada por el proceso de mejora continua, se detectó la existencia de oportunidades de mejora en la mayoría de los aspectos que se establecieron como determinantes para medir esta dimensión, los cuales deberían ser reforzados. Motivo por el cual la gerencia debe revisarlos, en virtud de la importancia que tiene el cumplimiento de estas etapas en la mejora continua, para lograr la calidad, incorporándolo al proceso de gestión.

\section{REFERENCIAS BIBLIOGRAFICAS}

Cantú, J. (2011). Desarrollo de una cultura de calidad. Segunda edición. Editorial McGrawHill Latinoamericana. México

Miranda, F. J., Chamorro, A., y Rubio, S. (2007). Introducción a la gestión de la calidad. Editorial Delta Publicaciones. Madrid, España

Norma ISO 9001:2008. (2008). Sistema de Gestión de la Calidad. Requisitos. Tercera Revisión. Diciembre 2008

Ley del Sistema Venezolano para la Calidad. Gaceta oficial № 3742

Udaondo, M. (2007). Gestión de calidad. Editorial Díaz de Santos. Madrid. España

Newell, M. (2004). Preguntas y respuestas sobre la gestión de calidad. Editorial gestión 2000. México

Norma ISO 9000:2005 (2006). Sistemas de Gestión de la Calidad. Fundamentos y vocabulario. Tercera revisión. Abril 2006 
Muñoz, J. (2009). La gestión integrada: calidad, seguridad y medio ambiente. Segunda edición. Editorial SERFOREM, S. L. España

Pérez, P. y Munera, F. (2007). Reflexiones para implementar un sistema de gestión de calidad (ISO 9001:2000) en cooperativas y empresas de economía solidaria Editorial Universidad Cooperativa de Colombia. Colombia

Deming, E. (1989). Calidad, productividad y competitividad. La salida de la crisis. Primera edición. Editorial Díaz de Santos. Madrid 